\title{
Sequential nonabsorbing microwave single-photon detector
}

\author{
Ivan Iakoupov $\odot,{ }^{*}$ Yuichiro Matsuzaki, ${ }^{\dagger}$ William J. Munro, and Shiro Saito \\ NTT Basic Research Laboratories, NTT Corporation, 3-1 Morinosato-Wakamiya, Atsugi, Kanagawa 243-0198, Japan
}

(Received 9 June 2019; accepted 10 July 2020; published 11 August 2020)

\begin{abstract}
We propose a nonabsorbing microwave single-photon detector that uses an artificial atom as a coherent interaction mediator between a traveling photon and a high- $Q$ resonator, fully exploiting the knowledge of the photon's arrival time. Our proposal can be implemented with the current level of technology and achieves distinguishability (probability of distinguishing between zero and 1 photon) in excess of $98 \%$ for realistic parameters. This is better than any of the similar detector proposals, even the ones using several artificial atoms.
\end{abstract}

DOI: 10.1103/PhysRevResearch.2.033238

\section{INTRODUCTION}

Efficiently detecting traveling microwave photons is an extremely challenging yet important task for future quantum technology. Currently, it is still not clear what is the best approach or whether there even is an approach that can be used in all situations. From the multitude of different proposals, we will restrict our attention here to the more versatile nonabsorbing detectors, so that detectors that absorb without reemitting or reemit the photon at a different frequency [1-9] are out of scope of this article. There is a number of recent proposals for the nonabsorbing microwave single-photon detectors [10-14] that can, in principle, be operated continuously, in the sense that the detector could be continuously interrogated for the presence of a photon. This way, both the presence of a photon and its arrival time could be obtained. The other type of the nonabsorbing detectors is the one that relies on knowledge of the photon's arrival time (or an arrival time window), and this is the approach taken in the recent experimental realizations $[15,16]$. We will call this type of the detectors sequential, since their principle of operation usually consists of a sequence of different operations, where the simplest version involves two distinct steps: (1) interaction between the incident photon and the detector and (2) interrogation of the detector. For instance, in Refs. $[15,16]$, step 1 is the controlled-phase gate between the photon and an artificial atom [17]. Often, trying to perform step 2 simultaneously with step 1 results in the detector not working at all, since interrogation will prevent the interaction. The continuous-mode detectors have various approaches to

\footnotetext{
*Present address: College of Liberal Arts and Sciences, Tokyo Medical and Dental University, 2-8-30 Kounodai, Ichikawa, Chiba 272-0827, Japan; ivan.las@tmd.ac.jp

${ }^{\dagger}$ Present address: Device Technology Research Institute, National Institute of Advanced Industrial Science and Technology (AIST), 1-1-1 Umezono, Tsukuba, Ibaraki 305-8568, Japan.

Published by the American Physical Society under the terms of the Creative Commons Attribution 4.0 International license. Further distribution of this work must maintain attribution to the author(s) and the published article's title, journal citation, and DOI.
}

make the interrogation during the interaction time be less detrimental [12-14].

The line between the continuous-mode and the sequential detectors is often blurred, however, as the continuous-mode detectors can also be operated sequentially where the sequence consists of "interrogation off" and "interrogation on." This is the mode that is used for the calculation of one of the often used figures of merit for the single-photon detectorsthe distinguishability (also known as "measurement fidelity"), which is the probability of correctly distinguishing between zero and one photon. Operationally, this is because the contribution of the signal due to an incident photon is finite, but the contribution of the (quantum and technical) noise can always be increased by making the interrogation time longer. Therefore, the best distinguishability value is obtained when the interrogation starts from the arrival time of the incident photon and continues until some later time that is determined by the response of the detector. In some cases, the proposals, which we call continuous-mode here, are not even analyzed as such, and only their sequential mode of operation is quantified by calculating the distinguishability $[12,13]$. Even for the cases when the quantitative continuous-mode analysis is done [14], the distinguishability is usually playing a very prominent role in the analysis. The reported distinguishabilities are in the range $70-96 \%$. Compared to those proposals, our proposed detector can no longer be operated in a continuous mode but achieves higher distinguishability with less experimental complexity (in terms of the number of artificial atoms).

In the telecom wavelengths, operation of the continuousmode detectors in the sequential mode (usually called the gated mode) is widely practiced in quantum communication $[18,19]$ to reduce noise [20]. In general, the photon's arrival time at the detector can be determined whenever the photon's emission time is known and the path length between the source and the detector is constant. Both assumptions hold true for a typical setup involving microwave photons and superconducting circuits, where deterministic single-photon sources are available [21,22], and the photons are routed by fixed transmission lines. Hence, sequential operation is usually possible, and our results suggest that a sequential detector that is designed to fully exploit the knowledge of the photon's arrival time is preferable over the 


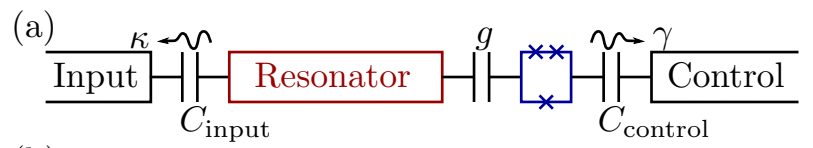

(b)

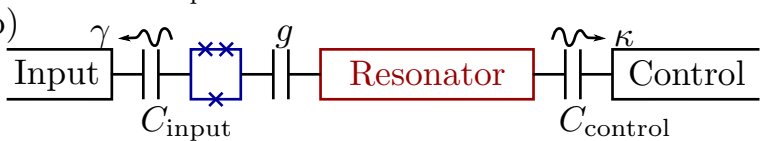

(c)

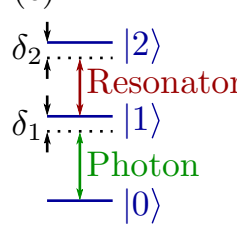

(d)

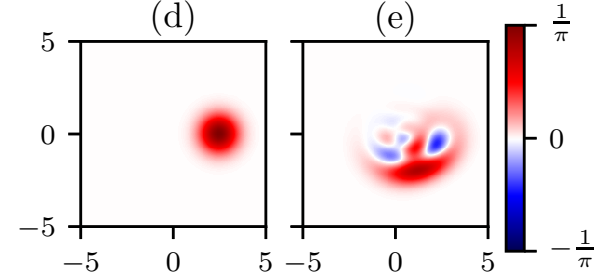

Reverse

(f)

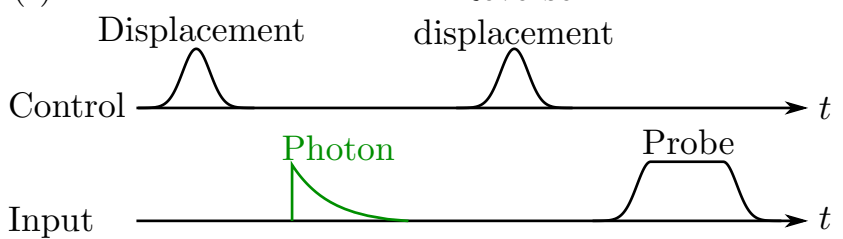

FIG. 1. (a) The usual microwave circuit QED setup. Here with a persistent-current artificial atom [27] and capacitive couplings, but many other variations are possible. The coupling capacitance $C_{\text {control }}$ is relatively small to prevent the artificial atom from decaying into the control transmission line (right). The coupling capacitance $C_{\text {input }}$ is relatively large to facilitate the interaction of the fields in the input transmission line (left) with the resonator. The decay rates are indicated next to the respective capacitive coupling that gives rise to them ( $\gamma$ means both decay rates $\gamma_{01}$ and $\gamma_{12}$ ). (b) The setup employed for our proposed detector. The coupling capacitances are the same, but the places and the roles of the qubit and the resonator are reversed. (c) The level diagram of the artificial atom and indication of the system that the given transition is coupled to (either the incident photon or the resonator). (d) Wigner function of the initial coherent state of the resonator. The average photon number is $\left\langle\hat{a}^{\dagger} \hat{a}\right\rangle=3$ [13]. (e) Wigner function of the resonator state (reduced density matrix) after the interaction with an incident photon with bandwidth $\gamma_{c} / \gamma_{01}=0.1$ for a time $T_{\text {interact }} \approx 92 / \gamma_{01}$. The other parameters are as follows: $\kappa / \gamma_{01}=3.2 \times 10^{-5}, \gamma_{11} / \gamma_{01}=$ $3.2 \times 10^{-3}, \gamma_{22} / \gamma_{01}=6.4 \times 10^{-3}, \gamma_{12} / \gamma_{01}=0.1, \delta_{1} / \gamma_{01} \approx-1.380$, $\delta_{2} / \gamma_{01} \approx-96.89, g / \gamma_{01} \approx 7 . T_{\text {interact }}, \delta_{1}$ and $\delta_{2}$ were chosen to minimize the error probability $P_{E, M}$, giving $P_{E, M} \approx P_{E, \text { opt }} \approx 2.2 \%$. (f) The proposed detection sequence for the setup in (b). The single-photon field is green and classical drives (displacements and probe) are black. They are incident from the two different transmission lines ("Control" and "Input").

continuous-mode detectors that are operated in the sequential mode.

We believe that our proposed detector could also be competitive against the existing experimental realizations of the sequential detectors $[15,16]$ where an artificial atom is used to store a state that is measured after an initial interaction with the incident photon, mediated by the resonator [see Fig. 1(a)]. The proposed detector uses the resonator to store a state that is measured after an initial interaction with the incident photon, mediated by the artificial atom [see Fig. 1(b)]. Thereby, our proposed detector reverses the roles of the artificial atom and the resonator. Technical details complicate the answer with respect to which approach is better (both give distinguishability $\mathcal{F}=100 \%$ in the idealized limit), but it is usually easier to achieve long coherence time in resonators [23-25] than in artificial atoms [26]. Therefore, our proposed detector may be less susceptible to the errors due to the finite coherence time of the stored state, which is one of the main sources of error $[15,16]$.

\section{SETUP}

The setup of our proposed detector is shown in Fig. 1(b). It consists of a three-level artificial atom and a resonator. The artificial atom is coupled both to an input transmission line and the resonator. From the input transmission line, a single photon is incident with a carrier frequency that is detuned from the $|0\rangle \leftrightarrow|1\rangle$ transition by $\delta_{1}$ [see Fig. 1(c)]. The resonator is close in frequency to the transition $|1\rangle \leftrightarrow|2\rangle$, resulting in a coupling $g$ with detuning $\delta_{2}$. The resonator frequency is assumed to be far detuned from the transition $|0\rangle \leftrightarrow|1\rangle$ such that the coupling is negligible. The resonator is also assumed to not directly couple to the input transmission line, e.g., due to suppression of the coupling by a Purcell filter [28] at the resonator frequency attached to the input transmission line [left in Fig. 1(b), not shown]. Depending on the bandwidth, the same or a different Purcell filter could also suppress the decay from the state $|2\rangle$ of the artificial atom, resulting in a small decay rate $\gamma_{12} / \gamma_{01}=0.1$ that was assumed in Ref. [13]. We keep the same $\gamma_{12} / \gamma_{01}$ for an easier comparison. The resonator is also coupled to a control transmission line with decay rate $\kappa$, making it possible to perform displacements of the resonator field.

The setup described above is very similar to the setup of Ref. [13], but with important differences, as described below. The Hamiltonian for our setup in the interaction picture with the rotating wave approximation made is

$$
H=\hbar \delta_{1} \hat{\sigma}_{11}+\hbar\left(\delta_{1}+\delta_{2}\right) \hat{\sigma}_{22}-i \hbar g\left(\hat{a} \hat{\sigma}_{21}-\hat{a}^{\dagger} \hat{\sigma}_{12}\right),
$$

where $\hat{\sigma}_{\mu \nu}=|\mu\rangle\langle\nu|$ are the atomic operators and $\hat{a}$ is the annihilation operator of the resonator. Compared to the Hamiltonian of Ref. [13], the above Hamiltonian does not have the term $-i \hbar E\left(\hat{a}-\hat{a}^{\dagger}\right)$ that describes the always-on drive of the resonator. This is because in our proposal, the Rabi frequency $E$ is set to zero during the entire detection sequence, except for the displacements that assume a large-enough $E$ for the displacements to happen in a time much shorter than any other timescale.

The incident photon that arrives from the input transmission line is modeled by a source resonator with decay rate $\gamma_{c}$ [11-14], setting the mode shape of the photon to be a decaying exponential in time. The corresponding master equation is [13]

$$
\begin{aligned}
\dot{\rho}=\mathcal{L} \rho= & -\frac{i}{\hbar}[H, \rho]+\gamma_{c} \mathcal{D}[\hat{c}] \rho+\gamma_{01} \mathcal{D}\left[\hat{\sigma}_{01}\right] \rho \\
& +\gamma_{12} \mathcal{D}\left[\hat{\sigma}_{12}\right] \rho+\kappa \mathcal{D}[\hat{a}] \rho \\
& +\sqrt{\gamma_{c} \gamma_{01}}\left(\left[\hat{c} \rho, \hat{\sigma}_{10}\right]+\left[\hat{\sigma}_{01}, \rho \hat{c}^{\dagger}\right]\right),
\end{aligned}
$$

where $\mathcal{D}[\hat{r}] \rho=\frac{1}{2}\left(2 \hat{r} \rho \hat{r}^{\dagger}-\rho \hat{r}^{\dagger} \hat{r}-\hat{r}^{\dagger} \hat{r} \rho\right), \hat{c}$ is the annihilation operator of the source resonator, and the terms proportional to 
$\sqrt{\gamma_{c} \gamma_{01}}$ describe the coupling of the source resonator to the artificial atom using the cascaded systems formalism [29,30]. Setting $\kappa$ to be much smaller than in Ref. [13] (which used $\left.\kappa / \gamma_{01}=0.037\right)$ is the key difference of our proposal. Ideally, $\kappa \rightarrow 0$ can be taken, corresponding to the limit of $\kappa$ being negligible on the time scales of the detection sequence. Such a choice of $\kappa$ prevents the continuous-mode operation (possible in the proposal of Ref. [13]), because the resonator state cannot be probed directly. Instead, we propose a detection sequence [Fig. 1(f)] whose final step is to probe the artificial atom to gain the information about the resonator state. Before we go in the details about this procedure, we can explain the high distinguishability of the detector by considering the distinguishability of the resonator states for the cases with and without an incident photon.

The detection sequence starts by initializing the artificial atom in state $|0\rangle$ and the resonator in a coherent state. The latter could be accomplished by initializing in a vacuum state and displacing it. We choose the average photon number $\left\langle\hat{a}^{\dagger} \hat{a}\right\rangle=3$ [13], resulting in the coherent state of Fig. 1(d). At this point, an incident photon will change the state into the one shown in Fig. 1(e) after an interaction time $T_{\text {interact }}$. We believe that the interaction is related to the selective number-dependent arbitrary phase (SNAP) gates [31], but with the difference that our scheme uses a single photon rather than a classical field to drive the interaction. If no photon is incident and $\kappa \rightarrow 0$, then the resonator state stays the same, because the coupling of the transition $|0\rangle \leftrightarrow|1\rangle$ of the artificial atom and the resonator is assumed to be negligible. For $\kappa>0$, the only change is a decay of the resonator photon number, i.e., a displacement toward the vacuum state. Since a complete displacement towards the vacuum state is part of the detection protocol (as explained below), the error due to this decay can be compensated for.

We use two different distinguishability measures for the reduced density matrices of the resonator, $\rho_{0}$ and $\rho_{1}$, that correspond to the vacuum and single-photon inputs, respectively. The first one is the distinguishability $\mathcal{F}_{\text {opt }}$ that uses an (unspecified) optimal measurement and assumes a 50/50 probability of the two states. It can be written $\mathcal{F}_{\text {opt }}=1-$ $P_{E, \text { opt }}$, where [32]

$$
P_{E, \mathrm{opt}}=\frac{1}{2}-\frac{1}{4}\left\|\rho_{0}-\rho_{1}\right\|_{\mathrm{tr}},
$$

with $\left\|\rho_{0}-\rho_{1}\right\|_{\text {tr }}$ being the trace norm of $\rho_{0}-\rho_{1}$. For the second distinguishability measure, we note that we can write $\rho_{0}=|\alpha\rangle\langle\alpha|$ for some coherent state $|\alpha\rangle$, which is either the initial state shown in Fig. 1(d) (for $\kappa \rightarrow 0$ ) or a displaced version of it (for $\kappa>0$ ). We define two measurement operators, $M_{0}=|\alpha\rangle\langle\alpha|$ and $M_{1}=I-M_{0}$, where $I$ is the identity operator. Then the distinguishability is $\mathcal{F}_{M}=1-P_{E, M}$, where [33]

$$
P_{E, M}=\frac{1}{2} \operatorname{tr}\left(\rho_{0} M_{1}\right)+\frac{1}{2} \operatorname{tr}\left(\rho_{1} M_{0}\right),
$$

again assuming a $50 / 50$ probability of the two states $\rho_{0}$ and $\rho_{1}$. This second state distinguishability measure is an idealization of the more realistic measurement procedure that we will discuss later. Since $M_{0}=\rho_{0}$, the term $\operatorname{tr}\left(\rho_{0} M_{1}\right)$ that represents the dark count probability in $P_{E, M}$ vanishes, resulting in $P_{E, M}=\operatorname{tr}\left(\rho_{1} M_{0}\right) / 2$.
To model the pure dephasing of the artificial atom, we add the terms $\gamma_{11} \mathcal{D}\left[\hat{\sigma}_{11}\right] \rho$ and $\gamma_{22} \mathcal{D}\left[\hat{\sigma}_{22}\right] \rho$ to the master equation (2) and set $\gamma_{22} / \gamma_{11}=2$ to account for the larger dephasing rate of the higher energy levels [34]. Assuming the decay rate $\gamma_{01}=2 \pi \times 10 \mathrm{MHz}$ and the pure dephasing time $T_{\phi, \mathrm{AA}}=10 \mu \mathrm{s}$ for the artificial atom, we have $\gamma_{11} / \gamma_{01}=$ $2 /\left(T_{\phi, \mathrm{AA}} \gamma_{01}\right) \approx 3.2 \times 10^{-3}$ and $\gamma_{22} / \gamma_{01} \approx 6.4 \times 10^{-3}$. For the resonator, we assume $T_{1, R}=500 \mu \mathrm{s}$ [35], giving $\kappa / \gamma_{01}=$ $1 /\left(T_{1, R} \gamma_{01}\right) \approx 3.2 \times 10^{-5}$. Even longer coherence times are possible with the $3 \mathrm{D}$ resonators [23-25]. For reference, we also compare to $T_{1, R}=50 \mu \mathrm{s}$ that represents the $2 \mathrm{D}$ resonators [36]. The other imperfections in the model are the nonzero ratio $\gamma_{c} / \gamma_{01}$ and the imperfect dispersive interaction. The latter depends on how close the Hamiltonian (1) is to the perfect dispersive Hamiltonian

$$
H_{\text {disp }}=\hbar \delta_{1} \hat{\sigma}_{11}-\hbar \chi \hat{\sigma}_{11} \hat{a}^{\dagger} \hat{a}
$$

where $\chi=g^{2} /\left(\delta_{1}+\delta_{2}\right)$. We choose $g=7 \gamma_{01}=2 \pi \times$ $70 \mathrm{MHz}$ [35].

Lossy elements like circulators in the path between the source and the detector can be described by fictitious beam splitters [12]. A beam splitter with the transmission coefficient $t$ corresponds to the power loss $1-t^{2}$. By using the beam splitter relations and tracing over the loss mode, the final state $\rho_{1}$ (single-photon input) is modified to $t^{2} \rho_{1}+\left(1-t^{2}\right) \rho_{0}$, while the final state $\rho_{0}$ (vacuum input) is left unchanged. The error probability $P_{E, M}$ with a lossy element can be calculated in a particularly simple way due to $M_{0}=\rho_{0}$ (perfect compensation for $\kappa>0)$. We get $P_{E, M}=\left(1-t^{2}\right) / 2+t^{2} \operatorname{tr}\left(\rho_{1} M_{0}\right) / 2$. For small power loss, we can approximately say that half of the power loss $1-t^{2}$ gets added to the intrinsic error probability of the detector.

\section{RESULTS}

In Fig. 2, we vary $\gamma_{c}$ and plot $P_{E, M}$ for optimal $\delta_{1}$ and $\delta_{2}$ using both Hamiltonians $H$ and $H_{\text {disp. }}$. We see that the optimal detunings are such that $\chi=g^{2} /\left(\delta_{1}+\delta_{2}\right) \approx-0.5 \gamma_{01}$ with a small change for the considered range of $\gamma_{c}$. This shows that the ratio between the decay rate of the artificial atom and the dispersive shift should be (approximately) fixed, similarly to Ref. [15], where the decay rate of the resonator and the dispersive shift needed to have a fixed ratio. Additionally, $\delta_{1} / \chi \approx 2.75$ for the considered range of $\gamma_{c}$. It is close to $\left\langle\hat{a}^{\dagger} \hat{a}\right\rangle=3$, which is the average number of photons in the resonator state. This shows that the primary function of the detuning $\delta_{1}$ is to compensate for the static shift of the frequency of the artificial atom due to the dispersive interaction $-\chi \hat{\sigma}_{11}\left\langle\hat{a}^{\dagger} \hat{a}\right\rangle$. The interaction time $T_{\text {interact }}$ is varied such that $T_{\text {interact }} \leqslant 10 / \gamma_{c}$ with the smallest $P_{E, M}$ is chosen. For example, for $\kappa / \gamma_{01}=3.2 \times 10^{-5}$ and $\gamma_{c} / \gamma_{01}=0.1, T_{\text {interact }} \approx$ $92 / \gamma_{01} \approx 1.5 \mu \mathrm{s}$ is chosen. For the optimized parameters, $P_{E, \text { opt }} \approx P_{E, M}$, which would have lead to completely overlapping curves in Fig. 2 (very small difference is visible for $\left.\kappa / \gamma_{01}=3.2 \times 10^{-4}\right)$. This is not always the case: If we used exactly the same parameters as in Ref. [13] except setting $\kappa=0\left(\gamma_{c} / \gamma_{01}=0.1, \gamma_{11}=\gamma_{22}=0, \delta_{1} / \gamma_{01}=-0.8\right.$, $\left.\delta_{2} / \gamma_{01}=-18, g / \gamma_{01}=2.45\right)$, then $P_{E, M} \approx 6.7 \%$ and $P_{E, \mathrm{opt}} \approx$ $4.6 \%$. 


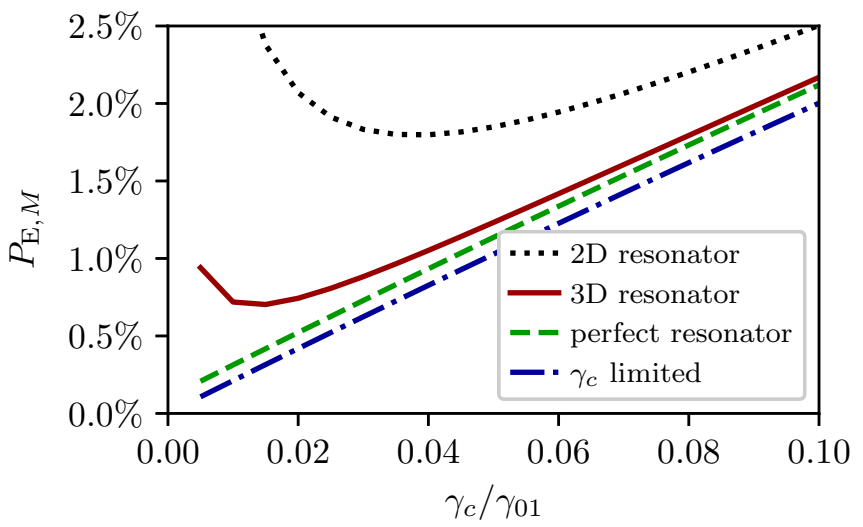

FIG. 2. Error probabilities $P_{E, M}$ as a function of the input photon bandwidth $\gamma_{c}$. Common parameters: $\gamma_{12} / \gamma_{01}=0.1, g / \gamma_{01}=7$. Unless noted otherwise, Hamiltonian (1) is used, and we set $\gamma_{11} / \gamma_{01}=$ $3.2 \times 10^{-3}$ and $\gamma_{22} / \gamma_{01}=6.4 \times 10^{-3}$. The legend indicates the different scenarios: "2D resonator" uses $\kappa=3.2 \times 10^{-4} \gamma_{01}\left(T_{1, R}=\right.$ $50 \mu \mathrm{s})$, "3D resonator" uses $\kappa=3.2 \times 10^{-5} \gamma_{01} \quad\left(T_{1, R}=500 \mu \mathrm{s}\right)$, "perfect resonator" uses $\kappa=0$, and " $\gamma_{c}$ limited" uses Hamiltonian (5) with $\kappa=\gamma_{11}=\gamma_{22}=0$. For each value of $\gamma_{c} / \gamma_{01}$, the parameters $\delta_{1}, \delta_{2}$ and $T_{\text {interact }}$ were chosen to minimize $P_{E, M}$.

The solid red curve in Fig. 2 shows our results with the most complete model of the imperfections that uses the Hamiltonian (1) with $\kappa / \gamma_{01}=3.2 \times 10^{-5}$. The states in Figs. $1(\mathrm{~d})$ and $1(\mathrm{e})$ correspond to $\gamma_{c} / \gamma_{01}=0.1$ on this curve. The dotted black and dashed green curves only differ from the solid red one in setting $\kappa / \gamma_{01}=3.2 \times 10^{-4}$ and $\kappa=0$, respectively. The dash-dotted blue curve represents the almost ideal case with $\kappa=\gamma_{11}=\gamma_{22}=0$ and uses the Hamiltonian (5) so that it is only limited by the nonzero ratio $\gamma_{c} / \gamma_{01}$, achieving $\mathcal{F}_{M}=100 \%$ for $\gamma_{c} / \gamma_{01} \rightarrow 0$. Comparison of these curves illustrates the different effect of the imperfections of the artificial atom and the resonator. The imperfections of the artificial atom add a constant term to $P_{E, M}$, since the artificial atom only acts as a mediator of the interaction and effectively stores every part of the incident photon only for a limited time. The nonzero $\kappa$ of the resonator, on the other hand, gives a larger error with smaller $\gamma_{c} / \gamma_{01}$, since the state has to be stored in the resonator for a longer time.

A realization of the measurement used for $\mathcal{F}_{M}$ starts with an unconditional displacement of $\rho_{0}$ and $\rho_{1}$ such that $\rho_{0}=$ $|\mathrm{vac}\rangle\langle\mathrm{vac}|$, where $|\mathrm{vac}\rangle$ is the vacuum state of the resonator, and the ideal measurement operators become $M_{0}=|\mathrm{vac}\rangle\langle\mathrm{vac}|$ and $M_{1}=I-M_{0}$. An implementation of these measurement operators can be done by driving the artificial atom by a continuous wave field with a frequency close to the transition $|0\rangle \leftrightarrow|1\rangle$ from the input transmission line (using a switch $[16,37]$ to change from the single-photon source) and doing homodyne detection of the reflected field. The idea is that the dispersive interaction of the artificial atom with the resonator [see Eq. (5)] shifts the frequency of the transition $|0\rangle \leftrightarrow|1\rangle$ only if the resonator is in a state different from vacuum. This conditional frequency shift of the transition $|0\rangle \leftrightarrow|1\rangle$ gives a conditional phase shift of the reflected probe field applied at a fixed frequency. The complete detection sequence is summarized in Fig. 1(f).
The probing of the artificial atom is described by an additional Hamiltonian term $\hbar \Omega\left(\hat{\sigma}_{01}+\hat{\sigma}_{10}\right)$, where $\Omega$ is the Rabi frequency of the continuous wave drive, and using the stochastic master equation [11-14]

$$
d \rho=\mathcal{L} \rho d t+\sqrt{\eta} d W(t) \mathcal{H}[\hat{O}] \rho,
$$

with $\mathcal{H}[\hat{r}] \rho=\hat{r} \rho+\rho \hat{r}^{\dagger}-\operatorname{tr}\left(\hat{r} \rho+\rho \hat{r}^{\dagger}\right) \rho, \hat{O}=e^{-i \phi} \sqrt{\gamma_{01}} \hat{\sigma}_{01}$, $\eta$ being the efficiency of the homodyne detection $(\eta=1$ unless noted otherwise), and $d W(t)$ being a Wiener process. Since the interaction time is long relative to the duration of the incident photon, the atom is negligibly different from being in state $|0\rangle$ at the beginning of probing. The initial system state for probing is thus taken to be a product state of the artificial atom being in state $|0\rangle$, and the resonator being in the state determined by the interaction (with and without an incident photon) and the reverse displacement.

The corresponding homodyne current is

$$
I_{n}(t)=\sqrt{\eta}\left\langle\hat{O}+\hat{O}^{\dagger}\right\rangle+d W(t) / d t,
$$

where the subscript $n$ can be 1 or 0 , indicating whether a photon was incident or not, respectively. To filter this homodyne current, we define $\bar{I}_{n}(t)=\left\langle\hat{O}+\hat{O}^{\dagger}\right\rangle$ where the expectation value uses the density matrix evolved with the deterministic master equation (2). The filtered integrated homodyne current is then

$$
S_{n}=\int_{T_{\text {interact }}}^{T_{\text {interact }}+T_{\text {probe }}} I_{n}(t) h(t) d t,
$$

where the filter is $h(t)=\left|\bar{I}_{0}(t)-\bar{I}_{1}(t)\right|$ and $T_{\text {probe }}$ is the probing time. We generate $N_{\text {tot }}=10^{4}$ trajectories for each of the cases $n=0$ and $n=1$, resulting in the integrals $S_{n, j}$, where $j$ is the trajectory index.

The distinguishability is then $\mathcal{F}_{M \text {,real }}=1-P_{E, M \text {,real }}$, where (for a $50 / 50$ probability of the two states)

$$
P_{E, M, \text { real }}=\frac{1}{2} \frac{N_{S_{0, j}>S_{\mathrm{thr}}}}{N_{\mathrm{tot}}}+\frac{1}{2} \frac{N_{S_{1, j}<S_{\mathrm{thr}}}}{N_{\mathrm{tot}}},
$$

with $N_{S_{0, j}>S_{\mathrm{thr}}}\left(N_{S_{1, j}<S_{\mathrm{thr}}}\right)$ being the number of the integrals $S_{0, j}$ $\left(S_{1, j}\right)$ that are above (below) the threshold $S_{\text {thr }}$. The threshold $S_{\text {thr }}$ is chosen such that $P_{E, M \text {,real }}$ is minimized. This definition assumes that, on average, the integrals $S_{0, j}$ are smaller than the integrals $S_{1, j}$. It is the case in Fig. 3 which corresponds to $\gamma_{c} / \gamma_{01}=0.1$ on the solid red curve in Fig. 2. The term $N_{S_{0, j}>S_{\text {thr }}} / N_{\text {tot }}$ in the definition of $P_{E, M \text {,real }}$ is the dark count probability. We find that for the threshold $S_{\text {thr }} \approx-93$ that minimizes $P_{E, M \text {,real }}$ in Fig. 3 , the term $N_{S_{0, j}>S_{\text {thr }}} / N_{\text {tot }}$ is negligible compared to $N_{S_{1, j}<S_{\text {thr }}} / N_{\text {tot }}$, and the resulting error probability $P_{E, M \text {,real }} \approx 2.4 \%$ is determined by the latter term. This implies that including the effect of lossy elements with power loss $1-t^{2}$ in the path between the source and the detector can be done in the same way as for $P_{E, M}$ with the resulting expression $P_{E, M \text {,real }} \approx\left(1-t^{2}\right) / 2+t^{2} N_{S_{1, j}<S_{\text {thr }}} /\left(2 N_{\text {tot }}\right)$.

The error probability $P_{E, M \text {,real }} \approx 2.4 \%$ is slightly larger than $P_{E, M} \approx 2.2 \%$ for the same interaction parameters. It might be possible to optimize the probing parameters (see caption of Fig. 3) further to make the difference smaller. One of these parameters is the probing time $T_{\text {probe }}=500 / \gamma_{01}$, which could be either increased to reduce the error probability or decreased to shorten the total detection time $T_{\text {interact }}+T_{\text {probe }}=$ $592 / \gamma_{01}=9.4 \mu \mathrm{s}$. Imperfect homodyne detection efficiency 


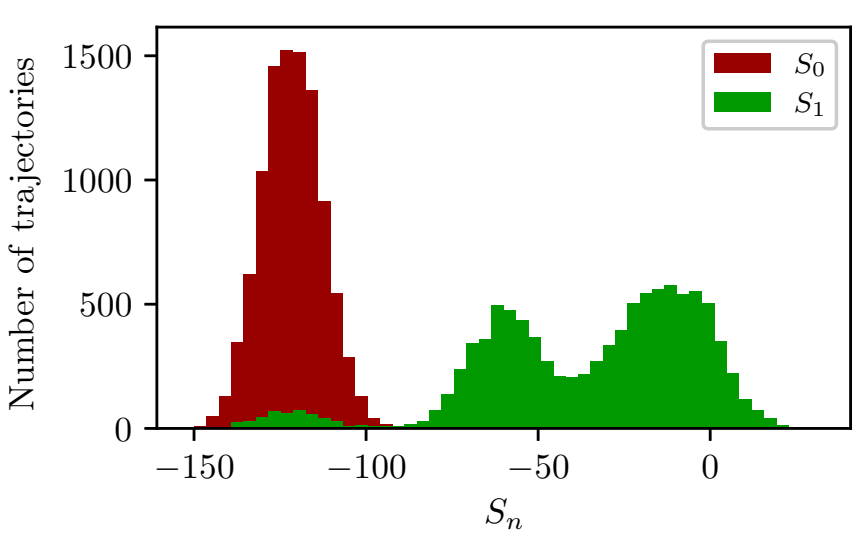

FIG. 3. Histogram of the filtered integrated homodyne currents, $S_{0}$ and $S_{1}$, that correspond to the case without and with an incident photon, respectively. The parameters correspond to $\gamma_{c} / \gamma_{01}=0.1$ on the solid red curve in Fig. $2\left(\kappa / \gamma_{01}=3.2 \times 10^{-5}, \gamma_{11} / \gamma_{01}=3.2 \times\right.$ $10^{-3}, \quad \gamma_{22} / \gamma_{01}=6.4 \times 10^{-3}, \quad \gamma_{12} / \gamma_{01}=0.1, g / \gamma_{01}=7, \quad \delta_{1} / \gamma_{01} \approx$ $\left.-1.380, \delta_{2} / \gamma_{01} \approx-96.89, T_{\text {interact }}=92 / \gamma_{01}\right)$. The parameters for probing of the artificial atom are: $\delta_{1}=0.1 \gamma_{01} \phi=\pi / 2, \Omega / \gamma_{01}=$ $0.2, T_{\text {probe }}=500 / \gamma_{01}$. The total number of the trajectories is $N_{\text {tot }}=$ $10^{4}$. The resulting error probability is $P_{E, M \text {,real }} \approx 2.4 \%$.

$\eta=0.5$ increases the error probability to $P_{E, M, \text { real }} \approx 3.1 \%$, showing that our proposal is robust against a nonunit $\eta$.

\section{DISCUSSION AND CONCLUSION}

The distinguishability of our sequential detector proposal compares favorably against the distinguishability reported for the continuous-mode detector proposals [12-14], even if they use several artificial atoms. Before the comparison, we note that all the considered setups are such that the photon to be detected is reflected back into the input transmission line. Therefore, a circulator is needed to prevent the reflected photon from going back to the source. Our proposal has the same behavior. In an experiment, the insertion loss of the circulator (around 4\% power loss [38]) will therefore affect the distinguishability, and we have explained above how such loss could be included in the theoretical analysis for our proposal. However, for an equivalent comparison with other proposals [12-14], we assume perfect circulators, since the distinguishability numbers with lossy circulators are not provided in all references.

As shown in Fig. 2, we report distinguishabilities $\mathcal{F}=$ 98\% and above for $\gamma_{c} / \gamma_{01} \leqslant 0.1$, which are better than the reported distinguishabilities in Refs. [12-14] (70-96\%). In the other proposals, the artificial atom decay rates may have a range of values [12], or the collective effects may make the single artificial atom rate less relevant than the decay rate of the entire ensemble [14]. In the former case [12], $\mathcal{F}=90 \%$ is reported for eight artificial atoms where $\gamma_{c} / \gamma_{01}$ varies between 0.5 and 0.1 for the different artificial atoms. In the latter case [14], $\gamma_{c} / \gamma_{B}=0.1$ is used, where $\gamma_{B}$ is the decay rate of the bright state of the ensemble, resulting in $\mathcal{F}=96 \%$ for four artificial atoms and one resonator. The proposal of Ref. [13] uses $\gamma_{c} / \gamma_{01}=0.1$ and reports $\mathcal{F}=90 \%$ for two artificial atoms and two resonators. Thus, our proposed detector achieves higher distinguishability with less experimental complexity (one artificial atom and one resonator). The difference in the distinguishability is even larger if we compare to a setup with the same experimental complexity as ours. The setup of Ref. [13] with one artificial atom, one resonator, and $\gamma_{c} / \gamma_{01}=$ 0.1 reports $\mathcal{F}=84 \%$ for $\eta=1$ and $\mathcal{F}=75.5 \%$ for $\eta=0.5$. With the same $\gamma_{c} / \gamma_{01}=0.1$, our setup achieves $\mathcal{F}=97.6 \%$ for $\eta=1$ and $\mathcal{F}=96.9 \%$ for $\eta=0.5$. This also shows that our setup is much more robust against a nonunit homodyne detection efficiency $\eta$ than Ref. [13].

In conclusion, we propose a nonabsorbing microwave single-photon detector with a larger distinguishability than in the similar proposals [12-14]. We accomplish this by completely removing the option of the continuous-mode operation (without the knowledge of the photon's arrival time) in the proposal of Ref. [13] and introducing a new detection sequence. Our proposal is different from the previous sequential setups $[15,16]$, as the roles of the artificial atom and the resonator are reversed such that the resonator is used as storage, instead of the artificial atom. This difference may make our proposal more attractive due to often better coherence properties of the resonators compared to the artificial atoms.

\section{ACKNOWLEDGMENTS}

We thank I. Mahboob and R. Budoyo for useful comments. This work was supported by CREST (JPMJCR1774), JST.
[1] G. Romero, J. J. García-Ripoll, and E. Solano, Phys. Rev. Lett. 102, 173602 (2009).

[2] Y.-F. Chen, D. Hover, S. Sendelbach, L. Maurer, S. T. Merkel, E. J. Pritchett, F. K. Wilhelm, and R. McDermott, Phys. Rev. Lett. 107, 217401 (2011).

[3] K. Koshino, K. Inomata, T. Yamamoto, and Y. Nakamura, Phys. Rev. Lett. 111, 153601 (2013).

[4] K. Koshino, K. Inomata, Z. Lin, Y. Nakamura, and T. Yamamoto, Phys. Rev. A 91, 043805 (2015).

[5] K. Inomata, Z. Lin, K. Koshino, W. D. Oliver, J.-S. Tsai, T. Yamamoto, and Y. Nakamura, Nat. Commun. 7, 12303 (2016).
[6] O. Kyriienko and A. S. Sørensen, Phys. Rev. Lett. 117, 140503 (2016).

[7] G. Oelsner, C. K. Andersen, M. Rehák, M. Schmelz, S. Anders, M. Grajcar, U. Hübner, K. Mølmer, and E. Il'ichev, Phys. Rev. Appl. 7, 014012 (2017).

[8] J. Leppäkangas, M. Marthaler, D. Hazra, S. Jebari, R. Albert, F. Blanchet, G. Johansson, and M. Hofheinz, Phys. Rev. A 97, 013855 (2018).

[9] R. Lescanne, S. Deléglise, E. Albertinale, U. Réglade, T. Capelle, E. Ivanov, T. Jacqmin, Z. Leghtas, and E. Flurin, Phys. Rev. X 10, 021038 (2020). 
[10] F. Helmer, M. Mariantoni, E. Solano, and F. Marquardt, Phys. Rev. A 79, 052115 (2009).

[11] B. Fan, A. F. Kockum, J. Combes, G. Johansson, I.-c. Hoi, C. M. Wilson, P. Delsing, G. J. Milburn, and T. M. Stace, Phys. Rev. Lett. 110, 053601 (2013).

[12] S. R. Sathyamoorthy, L. Tornberg, A. F. Kockum, B. Q. Baragiola, J. Combes, C. M. Wilson, T. M. Stace, and G. Johansson, Phys. Rev. Lett. 112, 093601 (2014).

[13] B. Fan, G. Johansson, J. Combes, G. J. Milburn, and T. M. Stace, Phys. Rev. B 90, 035132 (2014).

[14] B. Royer, A. L. Grimsmo, A. Choquette-Poitevin, and A. Blais, Phys. Rev. Lett. 120, 203602 (2018).

[15] S. Kono, K. Koshino, Y. Tabuchi, A. Noguchi, and Y. Nakamura, Nat. Phys. 14, 546 (2018).

[16] J.-C. Besse, S. Gasparinetti, M. C. Collodo, T. Walter, P. Kurpiers, M. Pechal, C. Eichler, and A. Wallraff, Phys. Rev. X 8, 021003 (2018).

[17] L.-M. Duan and H. J. Kimble, Phys. Rev. Lett. 92, 127902 (2004).

[18] N. Gisin, G. Ribordy, W. Tittel, and H. Zbinden, Rev. Mod. Phys. 74, 145 (2002).

[19] J. Zhang, M. A. Itzler, H. Zbinden, and J.-W. Pan, Light: Sci. Appl. 4, e286 (2015).

[20] G. Ribordy, J.-D. Gautier, H. Zbinden, and N. Gisin, Appl. Opt. 37, 2272 (1998).

[21] P. Forn-Díaz, C. W. Warren, C. W. S. Chang, A. M. Vadiraj, and C. M. Wilson, Phys. Rev. Appl. 8, 054015 (2017).

[22] Y. Zhou, Z. Peng, Y. Horiuchi, O. V. Astafiev, and J. S. Tsai, Phys. Rev. Appl. 13, 034007 (2020).

[23] M. Reagor, W. Pfaff, C. Axline, R. W. Heeres, N. Ofek, K. Sliwa, E. Holland, C. Wang, J. Blumoff, K. Chou, M. J. Hatridge, L. Frunzio, M. H. Devoret, L. Jiang, and R. J. Schoelkopf, Phys. Rev. B 94, 014506 (2016).

[24] C. Wang, Y. Y. Gao, P. Reinhold, R. W. Heeres, N. Ofek, K. Chou, C. Axline, M. Reagor, J. Blumoff, K. M. Sliwa, L. Frunzio, S. M. Girvin, L. Jiang, M. Mirrahimi, M. H. Devoret, and R. J. Schoelkopf, Science 352, 1087 (2016).
[25] A. Romanenko, R. Pilipenko, S. Zorzetti, D. Frolov, M. Awida, S. Belomestnykh, S. Posen, and A. Grassellino, Phys. Rev. Appl. 13, 034032 (2020).

[26] J. J. Burnett, A. Bengtsson, M. Scigliuzzo, D. Niepce, M. Kudra, P. Delsing, and J. Bylander, npj Quant. Info. 5, 54 (2019).

[27] T. P. Orlando, J. E. Mooij, L. Tian, C. H. van der Wal, L. S. Levitov, S. Lloyd, and J. J. Mazo, Phys. Rev. B 60, 15398 (1999).

[28] M. D. Reed, B. R. Johnson, A. A. Houck, L. DiCarlo, J. M. Chow, D. I. Schuster, L. Frunzio, and R. J. Schoelkopf, Appl. Phys. Lett. 96, 203110 (2010).

[29] C. W. Gardiner, Phys. Rev. Lett. 70, 2269 (1993).

[30] H. J. Carmichael, Phys. Rev. Lett. 70, 2273 (1993).

[31] R. W. Heeres, B. Vlastakis, E. Holland, S. Krastanov, V. V. Albert, L. Frunzio, L. Jiang, and R. J. Schoelkopf, Phys. Rev. Lett. 115, 137002 (2015).

[32] C. A. Fuchs and J. van de Graaf, IEEE Trans. Inf. Theory 45, 1216 (1999).

[33] J. M. Geremia, Phys. Rev. A 70, 062303 (2004).

[34] M. J. Peterer, S. J. Bader, X. Jin, F. Yan, A. Kamal, T. J. Gudmundsen, P. J. Leek, T. P. Orlando, W. D. Oliver, and S. Gustavsson, Phys. Rev. Lett. 114, 010501 (2015).

[35] C. J. Axline, L. D. Burkhart, W. Pfaff, M. Zhang, K. Chou, P. Campagne-Ibarcq, P. Reinhold, L. Frunzio, S. M. Girvin, L. Jiang, M. H. Devoret, and R. J. Schoelkopf, Nat. Phys. 14, 705 (2018).

[36] A. Megrant, C. Neill, R. Barends, B. Chiaro, Y. Chen, L. Feigl, J. Kelly, E. Lucero, M. Mariantoni, P. J. J. O’Malley, D. Sank, A. Vainsencher, J. Wenner, T. C. White, Y. Yin, J. Zhao, C. J. Palmstrøm, J. M. Martinis, and A. N. Cleland, Appl. Phys. Lett. 100, 113510 (2012).

[37] M. Pechal, J.-C. Besse, M. Mondal, M. Oppliger, S. Gasparinetti, and A. Wallraff, Phys. Rev. Appl. 6, 024009 (2016).

[38] See datasheets of circulators at, e.g., https://www. lownoisefactory.com/. 